pères, dont une bien faible partie seulement, à la vérité, est wattée. Toutefois, au bout d'un instant, sous l'effet de l'hystérésis et des courants de Foucault, le fer du transformateur s'échaufferait tellement que les isolements et l'enroulement secondaire seraient vite détériorés.

Moyennant cette simple précaution, cet appareil, contrairement au shunt à circulation d'eau, est véritablement, pour les courants alternatifs, un instrument pratique, à la tois de service et de contrôle. Il a le grand avantage de pouvoir fonctionner, comme nous l'avons déjà dit, avec tous les ampèremètres, de sorte que l'on peut utiliser un instrument de précision pour les vérifications, et se contenter en service courant d'un ampèremètre ordinaire, plus robuste et moins coûteux.

$$
\begin{gathered}
\text { C. LimB } \\
\text { Docteur ès-Sciences, Ingéneur-Electicien. }
\end{gathered}
$$

\section{LES APPAREILS DE MESURE ACTUELS}

Communication prísentée au Congrés de Marselle, par M. A. Durand, Chef de travaux au Laboratorre Contral d'Electricité

Depuis l'annce 1900 , on ne peut signaler comme appareils de mesures nouveaux que les uattmetres them'ques, dont les indications sont presque indépendantes de la fréquence et de la forme de la courbe du courant. Les efforts des constructeurs se sont surtoul bornéls à perfectionner les autres apparells : c'est amsı que, pour les voltmètres et ampèremètres à almant et cadre mobile, par exemple, on a, par l'emplor des alliages constantan et manganın, dimınué, dans une grande proportıon, l'erreur due aux variations de la temperature ambiante ; mass s, pour les courants continus, on parait arriver à un degré de précision suffisant pour les mesures industrielles, ll n'en est pas de mème pour les courants allermatifs, où l'emploi de tensions et d'mntensités de plus en plus élevées nécessite des transformateurs trop souvent mal éludiés, et qui sont, alors, la cause d'erreurs nouvelles.

Tout le mal, il faut bien le dire, n'est pas imputable au conistructeur, mass trop souvent dú à un mauvals emplos de l'mstrument. Beaucoup d'appareils, livrés exacts, ont leurs indications laussées, temporarrement ou définitivement, par l'influence des courants passant dans les conducteurs voisins.

Mon but, dans ce qui va suivre, n'est pas d'entrer dans les détails de construction, mais, par l'examen des défauts (1) et qualités des différents types d'appareils, de montrer quelle confiance nous pouvons avoir en leurs indications.

\section{I. - CONSIDÉRATIONS SUR LES APPAREILS DE MESURE}

Ln examinant les causes d'mcertitude, nous voyons que quelques-unes sont génćrales et peuvent affecter tous les apparells ; nous commencerons donc par les étudier. Ce sont : $1^{\circ}$ Les défauts de graduation, les erreurs de lecture (parallaxe) ; et $2^{\circ}$ Les actions mécaniques (pıvotage, variation des ressorts, amortassement).

D'autres, au conlraire, sont d'ordre électrique et seront signalés pour chaque type d'apparell ; elles sont dues à l'hystérésis, aux cuurants de Foucault, à lafiaiblissement des aumants, influence des champs magnétiques et électrostatıques, à la forme de courbe, ¿l la fréquence du courant, à la varıation des résistances des circuits avec la lempérature, au couples thermo-electronues, etc.

Graduations. - Les dilatations, dues aux variations hysroméliques de l'air, font restreindre l'emplos du carton pour les cadrans des appareils de mesure; les divisions sont maintenant, ou gravées sur métal, ou, le plus souvent, tracées sur du papier adherent à des feuilles métalliques.

(1) Beaucoup de ces défants constatéc, depuis plusieurs années, au Laboraloire Central d'Electricité, ont été signalés aussi dans différềtes publications et notamment par MM Armagnat. Instrumenls et methodes de mesures ; EDGGUMBE FT PUNGA, Journal of the Institution of Electrical Enaineers (19)4-1905); Rosa. Proceedings of the A merican Institule of Eleclrical Enqineer's (1905); II EINRICH ET BERCOWITZ. Handbuch der Electrolecknik, Zweiler Band funfle ableilaing.
Les erreurs de graduation seruient been fabbles, si les divistong étalent égales sur toute l'étendue de l'écholle; cetle dernèro condi. tion est rarement remplıe; aussi, la plupart des consluucteurs sont-ls obligés de graduer leurs appareils de 10 cn 10 par exemple, et de diviser ensurte ces intervalles aussi bren que porsible ; mais, à ces causes d'erreurs, vient s'ajouter le clingentent de forme de la courbe détalonnement dù alu jeu pris par les vis sous l'influence, et de la chaleur, et des vibrations (surtout en alternalif).

Dans l'état normal, on peut craindre pour la gradualıon dis appareils étalons, des erreurs absolues allesgnant 2 milliemes de la graduation maximum ; l'erreur relative me sera donc pas cons. tante sur toute l'échelle.

Erreurs de parallase. - Cetle erreur est fortement dmunurn dans certans instruments, grace au mroir placé sous l'aigulle. Le plan vertical de visée est ansi clétermmé par la superposttun de l'aiguille et de son image.

Ressors anlagonistes. - Les ressortis, en général en allages non magnétıques, láissent souvent beaucoup à dósırer ; ils jrí. sentent une viscosité élastrque qui se manifeste par un déplapo. ment du zéro, lorsque l'appareil reste en circuit un certan templs. Pour remédier à cet inconvénent, les constructeurs se servell souvent, comme couple antagonisle, de deux ressorts spmranx agissant en sens inverse, mass cette compensation n'esl pas par. faite, les deux ressorts ne travallant pas de la mome farcon. Il n'est pas rare de volr l'arguille dévier du 1/190 de l'échille maximum, après une mise en service d'une heure aux 8 dixiènts de cette échelle.

Une autre cause d'crreur provient aussi de la diminution dul couple des ressorts avec l'élévation de température.

C'est pour remédier au déplacement du zéro, dù à l'mégalití dans les ressorts, que les constructeurs (surtout à l'étranger) munissent les apparells de vis de rappel : ce dispositif serait it généralıser.

Suspension des équipages mobiles. - $1^{\circ}$ Suspuension par fil métallıque ou non. Employée dans le cas où la force agissanle est très fauble, cette suspension donne heu à un zéro mal dide. miné ; pour élimmer cetle erreur due à la viscosité du fil, il est to plus souvent nécessaire de faire des lectures en inversant le stens de la déviation; aussi ne doit-on compter que sur une approxim. tion de 0,5 pour 100 de la valeur maximum;

20 Suspension par les ressorts spiraux : employée par lorl Kelvin.

$3^{\circ}$ Suspension par couteaux appnyés sur des surfaces en forme de V (électromètre Kelvin);

$1^{\circ}$ Suspension sur pivots. Les pivots doivent être partıculièn. ment solgnicis pour me pas être une cause d'indécisıon dans les lec. tures ; lls reposent en général, sur des pierres dures montées, quelquefois sur ressorts ; ce dernier dispositif a pour but d'anor. trr les choes. Ciest aussi pour ménager les pointes des prots que les constructeurs font leurs équipages moblles le plus lóger possible.

L'indécision provenant des défauts de pivotage peut attendre, pour les bons appareils étalons, 2 millièmes de la déviatıon totale; cette inducision sera plus grande pour les appareils de tableau qu, souvent, ont une ajguille très longue. Lindécision dépendra, auss, de la position de l'angulle (horrzontale ou verticale); cette demire disposition étant la plus désavantageuse.

Amortissement. - Les constructeurs devraient apporter une grande attention à une question aussi importante que colle de lamortissement ; ll est, en effet, inadmissible de vorr, encore actuellement, les lectures aux apparells rendues impossibles par les oscillations de l'alguille. Il m'est arrivé, pour des amperre. mètres mis brusquement en crrcuit, sur du courant continu fourni par des accumulateurs, de compter 75 oscillations simples aroul l'arrêt de l'arguille ; la durée lotale pour avoir le régime stable sitail de 45 secondes ! Un tel apparell sera d'autant moins utilisable qu'aux variations de l'mtensilé du courant viemdront s'ajotler les perturbations dủes à la résonance qui pourra se prolure entre la périote d'oscillation de l'aiguille et celle du moleur.

Il est ì noter, que, quelque soit le genre damortisseur, l'action de la résistance de lair sur langulle indicatrice pourra sc faile sentir (notamment pour les grandes aiguilles).

Un grand amorhissement donnera lrop d'importance aux défauls de pivolage ; j'estime que deux oscillalions simples suffisent pour les apprareils étalons et de tableau, avec une durée totale d'osul. lations de 2 secondes. 
Lumortisscument des oscillations de liviquipage mobile eat Lhtenu : 10 par frottement intermittent (balances, électromètres Kelvin); $2^{\circ}$ Par frottement d'une palette dans un liquide (cau et glycérne, hule) ; l'hule employée dans certains apparenls peut donner lieu ì des erleuns grossiercs (l'hulle par l'abaissement do fompéralure devonant visqueuse); 30 Par frotlement d'une palette ou d'un piston dans me chambre à arr ; ce demer procédé, avec une bome construction, donne d'excellents résultats ; $4^{\circ}$ Par les counts de Foucault induits dans une pièce métallıque passant entre les poles d'un aimant.

\section{II. - EXAMEN DES DIFFÉRENTS TYPES D'INSTRUMENTS POUR TABLEAUX ET BOITES DE CONTROLE}

Appareils à cadre mobile et aimant. - Ces appareils, basés sur l'action d'un aimant sur le courant passant dans une bobine nobile, sont connus en France sous le nom de Deprez-d'Arsonval; us dérivent du Siphon recorder de lord Kelvin. Le modèle unduslicl a été créé par Weston.

Tls se composent, outre le boîtıer, de cinq prèces princıpales quı duivent, pour la certitude des indications, répondre a difiérentes condhlons ; ces plèces sont : Latmant, les surfaces polaires, le noyau en fer doux, le cadre mobile el sa résistance en série, et culin les ressorts anlagonisles qui servent à amener le courant dans le système moble.

dimants permanents. - Les aimants permanents utılisés dans les appareils de mesure doivent permettre de réaliser un champ mlense avec une grande stabihté. Pour arriver à ce résultat ll fiul que le champ démagnétısant soit assez faible. Il faut donc tr tris fables entrefers. De plus la répartition du flux devant être unforme dans un certain espace, la surface de cet entrefer doit Hre asse grande, d'où la nécessité do munir les amants de plèces polatres ou d'épanousssements polarres. A forme extérieure idenIque, la stabiluté est d'autant pliss grande, comme l'a montré Vadame Curle, que la force coercitive est plus grande; mass il ne foul pas oubher que, comme on a besoin d'un champ assez intense, ll faut aussi que le métal employé alt une perméabllité asse\% élevée.

Il en résulte qu'll vaut mieux employer les aciers au tungsène que les acicrs au molybdène. Ces derniers ont une force coercitive plus élevée que les premiers, mass une perméabilité plus farble. lien entendu ces aciers sont utılısés à l'état trempé. Le choix de In tempórature de trempe n'est pas indifférent. Dans certains cas les proprićtés magnétıques dépendent de la température de trempes; d'autre part il faut éviter à la trempe la formation de tapures.

Denx raisons infuent sur la constance des aimants permanents : $1^{\circ}$ Le métal trempé, aimanté, voit ses propriétés se modifier lentement. C'est le phénomène connu sous le nom de revenu après la irempe. Pour éviter ces varıations lentes, on peut provoquer un revenu rapide par une chauffe de 100 à 200 heures à $55^{\circ}$. : In certam nombre de causes font varier l'intensité d'aimantalion d'un aimant permanent; tels sont les chocs, l'action des champs extérieurs, les variations cycliques de température. Ame Curie a montré que toutes ces causes sont à peu près équivalentes. de telle sorte que lorsque l'une a agi, les autres sont snns action. Ainsi, d'après Mme Curie, il suffirait, après avoir aimanlé à saturation un aimant permanent, de le désaimanter du dixiòme de son aimantation rémanente, pour le rendre insensible i) la plupart des canses de variations auxquelles il peut itre soumis.

Mais 1 ne faut pas oublier que toute cause susceptible de faire varier l'intensıté d'aimantation rémanente de l'aimant sera suivie l'une pérıde de variation lente. Ainsi, comme l'a signalé Klemenric. il suffit rle placer un aimant dans une enveloppe métallique pour rue sa nouvelle intensitél d'amantation varie pendant un crrtain temps. Méme lorsque l'aimant est devenu constant, les vinrations de température entrainent une variation réversible de lintensité rl'aimantation. Cette variation est très compliquée romme l'a montrée Ainsworth. Elle peut etre pasitive ou négative. lille dirend non seulement du métal, mais de la température de trempe ol de la forme de l'aimant. Des recherches faites à la Reichanslalt sur des aimants de compteur en forme de fer à rheval entre $0^{\circ}$ et $60^{\circ}$, ont montuél lorsque la température augmentait, me rlminution du champ dans l'entrefer de $2 / 10000$ par degré. Mnusinn et Kennely (*) ont indiqué que, pour qu'un aimant fût

()) Houston et kennelt, Eclairage éleclrique, t. 1V, p. 36. stable il fallait que le rappout $\frac{L S}{v L S}$ fúl le plus pelit possible, l. et $S$ étant la longueur et la section de l'entrefer, et $s$ la longueur et la section du circuit magnélque, $v$ la force coercitivi du métal. l'après Hemich, la conditun que tous les constricteurs cherchent à remplr est :

$$
\frac{L s}{l s}<\frac{1}{100}
$$

Signalons pour terminer, que Plerce et Campbell ont préconsé l'emplos de la fonle trempée pour les ammants permanents ; que Maurain a indiqué qu'on pourrat peut-etre ubliser le fer éleciroly. tıque déposé dans un champ magnétique, el que Guillet a signalé les avantages de la trempe sous pression. Aucune de ces idées n’a, Jusqu'ici, reçu d'application à notre cormaissance.

Noyau en fer doux et masses polatres. - La proportionnalite de loute l'échelle dépend de ces prèces en fer doux. Leurs surfaces solgneusement travallées dolvent avour un centrage parfait et absolument indétréglable.

Queques constructeurs, Chauvn et Arnoux par exemple, pour simphifier la fabrication, supprinient les masses populares ; il he semble pas en résulter une infémorité bien grande au point de vue de l'exactitude des indicahons. D'autre part, dans les appareils à cadre Meylan-d'Arsonval, construits par la Compagnie nour la fabrication des compleurs, al n'existe pas de noyau en fer doux (").

cadre mobnle. - Le cadre mobile est constutué par un fil de culvre isolé, enroulé génćralement sur un cadre de cuivre; ce. carlre selt d'amortisseur pour les courants de Foucault. L'emplor d'un enroulement en cuivre permet, pour le meme nombre d'an. pères-tours, d'éviter un dégagement de chaleur suscrptible do déformer le cadre; par contre, la résistance électruque de l'en:oulement varjera avec la température de $4 / 1000^{\mathrm{e}}$ par degre cont. grade.

En sérle avec le cadre moblle, se trouve une résistance en manganine ; elle sert pour le réglage, et permet d'amener l'aigunle au maximum de déviation pour la différence de polentiel donl on dispose. Le coefficient de température du galvanomètre dépendra donc du rapport entre la valeur de la résistance totale de l'nppareil et celle de l'enroulement seul.

Poids de léquipage mobile. - Les constructeurs cherchent it diminuer le plus possible le poids de l'́quipage, afm de presorver les pointes des pivots; ce poids varie de 1 à 6 grammes.

Le Tableau cl-jomt, emprunté à louvrage dHenrich, montre Ia variation des constantes des millivolmetre's Weston el Siemen, selon les diffárentes périodes de fabrication.

\begin{tabular}{|c|c|c|c|c|c|c|c|}
\hline CONSTRUGTLLRS & ANNEE & $\frac{\text { CADRE }}{\begin{array}{c}\text { Po.ds } \\
\text { gldmmes }\end{array}}$ & $\frac{\text { MOBLLE }}{\text { Conpe E }}$ & 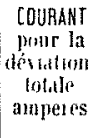 & $\begin{array}{c}\text { Anlible } \\
\text { poums } \\
\text { A }\end{array}$ & $\partial \frac{F}{p}$ & $\frac{v}{r \times A}$ \\
\hline Siemens \& Halshe & $1895-1906$ & 10,9 & 0,7 & 0,075 & 1,34 & 0,07 & 0,15 \\
\hline D) & 1907 & 0.9 & 0.08 & 0.015 & 0,40 & 0,09 & 0,10 \\
\hline Weston? & 1888 & 10 & 10 & 0,05 & 1.25 & 0,10 & 0,08 \\
\hline " & 1891 & $f i, \bar{T}$ & 0,90 & 0,10 & $1,2 \pi$ & 0.14 & 0,11 \\
\hline$n$ & 1893 & 3,7 & 0,40 & 0,03 & 0.4 & 0,11 & 0,28 \\
\hline$\eta$ & 1894 & $\mathbb{2}, 3$ & 0,10 & 0.02 & 0,4 & 0,18 & 0,45 \\
\hline$n$ & 1903 & 2 & 0,10 & 0,03 & 0,25 & 0,20 & 0,80 \\
\hline
\end{tabular}

Ressorts anlagonisles. - Les ressorts servent aussi ì amener le courant au cadre moble ; outre les qualités mócaniques, dont il a été question plus haut, ils devront avoir une faible résistance élechrique et la variation de celte résistance avec la tempiratiuc doit être fauble. Ces ressorts en bronze, a compostion plus ou mons secrète, ont souvent, un coefficient de température de $11 n$ millième par degré centigrade.

Couple des ressorts. - Ce couple est variable, naturellement, avec chacque constructeur, et dolt ctre different aussi, selon les types d'appareil. D'après Heinrich, le couple, en grammes-centimitre, nócessare pour produire une devalion de $90^{\circ}$ ne doit pas etre inférieur an vinglième du poids de la bobme mobile.

- (') Dans ces apparesls, un senl cole du cadre est arifi, et se deplace entre les surfaces polaures de l'aimant. 
la structure de l'allage el par conscyucnt, une vartation dans sa risistance électrique ; aussi ne devrait-on pas, it mon avis, depasser la température de $100^{\circ}$ pour le courant maxmmum. Il cst muspensoble, daus tous les cas, que le shmbl pusse supporter santi altération la courant maximum. Bealucoup de shunls no remplissent pas o die condihon.

Surfuces de conlact. Serrages. - Les surfaccs de contacl cnire las eronducleurs et les màchoires des sliunts sont, en général, trop

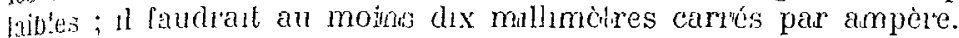
Lis boukns et les vis en fer ont l'moonvénent de sa rounler

Montage des shunts. - Le montage sur les tableaux est très mportants et, pour ne pas exagérer les couples thermo-ilechlques, il est nécessare d'avon' des serrages parfauts.
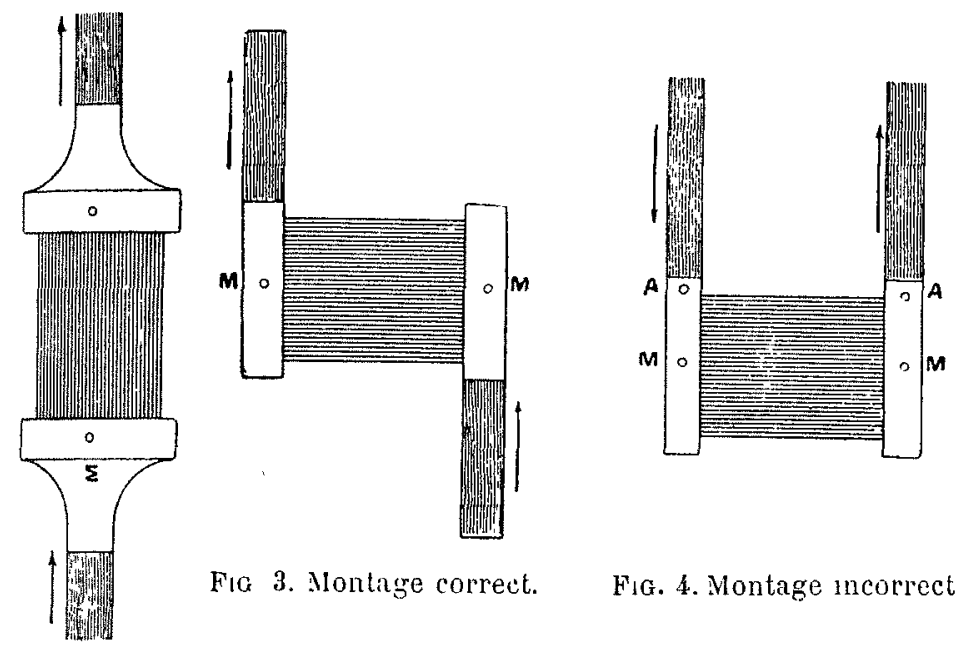

FIG 3. Montage correct.

Fig. 4. Montage incorrect

La position des cables amenant le courant n'est pas indifírente. pour les shunts de plusieurs nullers d'ampères l'inégale répartitiun des courantis peu produre des erreurs altelgnant 10 pour 100. té eliet se manifeste surtrut quant les prises de dérivation sont, no: at muleu, mais aux extrêmités des machorres. La figurue 3 represente des montages corrects ; la figure 4 un montage defectueux.

Précasion des ampèremètres. - On ne peut guère compter, pour rebalonnement des shunts, sur une erreur relatrve de moms de 5 millièmes surtout pour les fortes mtensités ; et, citant données loutes les causers d'errouns que nous avons énumórées pour les; mullivoltmètres, on voit qu'll faudra prendre de grandes précalltolos pour etre sur d'une exactitude re 1 pour 100 dans la mesure des intensités.

Voltmètres a CADRE Moblue, - Aucune difficulté speciale ricst i signaler pour les voltmètres ; il osl sculement essentrel davor des rosistances de section suffisante pour rester en crrcut sans dévation de température exagérée et sans variation dans leurs valeurs. La résistance des circults varle de 100 à 200 olms par volt ; deus ces conditions, le coefficuent de tempórature cst négligeable pour tous les voltmètres a partir de 30 volts, car la résıstanca du fil du cadre (en cuivre) est faible devant la résistance de réglage. Les voltmètres sont, a tous les pomts de vue, buen supérieurs aux ampèremètres à shunt.

Réglagle des voltmètres. - Les divisions etant tracécs sur le papuer, les constructeuns règlent dófinitivement les apparells. sout en fassant vamer la résistance mise en séme avec lo cadre, sont au moyon d'un shunt magnétique ; ce dernier procédé, très employé, à lavantage d'étalonner aussi le voltmètre en milli-ampèremètre, car il permet de donner ì la résistance de lapparenl un rapport très sample avec la dafférence de potenticl aux borncs.

Bortes de controle. - Ces bo:tes renferment un voltmètne et un ampèremètre (à cardre mobile) ì sensibllite multiples. Elles sont destinées à vérufier les mstallations ; aussi comportent-elles des appareils plus précis que ceux des tableaux de distribution. On a cherché, pour cas boites, à rédurre le polds ders appareils. de sorte que, le plus souvent, ll en résulte l'mpossibilıté de lassser l'apparell cn crrcuit, d'une façon continue, au maximum de la charge. Les shunts sont souvent montés de telle sorte qu'ils pusssent etre changés sans couper le courant. Les apparells sont fixés sur une planchetle, et il n'est pas indifférent (pour less raisons indiquées, a propos des aimants et de l'influence des champis magnitiques), de changer de place les appareils ou de mettre la planchelte dans le voisinage de plèces de fer.
Je signale de nouveau, lout particulierement, la baite de cuntroble Wieston, dont l'étalonnement peut dre vérulié sur place, glace au disposilif potentiométrique cu'elle comporte.

Appareils a fer doux, cadre mobile et aimant. - Au milicu du cadre d'un galvanomètre lieprez-d'Al'sonval se trouve une petite palette en fer doux s'orientant daus le sens des lignes de force de l'amant ; la force duredruce est ansi due aux ressurls et a l'adion de l'amant sur cetle palette de fer doux.

Ces appareils etudrós par $\lambda$. Weiss () sont très intiressants en ce sens que, dans certames limiles, leurs molications sont indépendantes des variatıons de l'aumant.

Appareils à fer doux et aimants, type Deprez-Garpentier. - Ces apprareils, très en vogue il y a qunne ans, sont actuellement presque totalement abandonmés. Les systemes mobiles avaent cependant l'avantage d'etre tris ancirodique, el de prondre instantan'ment leur posilion dépulubre ; par contre, il lenr fallan. une force electromagnestupe considerable, neccisitant un grand nombre d'ampères-tours ; ll éalt done mpossıble de lausser les voltmétres en curcult, cal l'intensité du comant élat sulfisanmenl, elevée pour faure varter, avec la résislance des enroulements (en curve), les indıcations de l'apparell Mass re qui a surtout contribur a la disparition de ce type d'anparenls, cest la disamantalion de lamant qu causal frúquemment aux molualoms des avances de 15 à 20 pour 100 . Celte dórammantation clat flue à $1 \mathrm{~m}$ Huence des conclucteurs vorsins, et notamment, dans lauripiremètre, à l'action sur l'aimant des ampères-tours nécrssaires all fonctronnement de l'appareil ; il faut remarquer, on omlo, qu"en cas de court-curcuit les amperes-tours peuvent devernr comsulirables.

Appareils à fer doux sans aimant. - Ces apparells, cxibinrment nombreux conme formes, sont très employés ; lls ont lavantage d'etre très robustes, el de pouvonr servil en couranl ailernahf. Leur pmix do vente, très modique, est canse de leur maturasse construolion.

Le couple électromagnétique est dù a laction d'un solemorite sur une ou plusieurs pièces en fer qui sunt altrées on repoussios Le couple antagoniste est produrt par des ressuris ou far far vesanteur ; dans ce dermer cas, il est absolmment nécessamro d'unstaller l'appareil de telle sorte que l'agulle sol au \% cro quand 11 ne passe aucun courant.

Les indications de ces instruments sont faussies par l'hystérésis, les champs magnétiques extérıeurs, la frétuence.

IIystérésis. - En couraut contmu, lhystérésis se manifeste par la différence des indications de l'apparell selon que le courant est croissant ou décrorssant ; pour dnminuer cetle action, il faul, rue les lignes de force dams le fer solent courtes, el que le 1er solt, ou saturé, on an contranro peu aimanté : cuns le premier cius, le mobile sela léger, dans le second sl sera lourd (pour les memes ampères-tours). Cetle dernière solution est gannéraltiment adojotíe, car, d'après certams constructeurs, olle a l'avantage do peinethre de fare con courant contmu I b́lalomement dapparels servan, en courant alternaluf ; en effel, si le for est sulmu, la force est, sensublement proportionnelle a la valeur instanlance du courant, et l'instrument indique la valeur moyome; landis que si l'nduction est fable, la force étant scusiblemenl proportionnelle an carré de l'intensité, l'apparell indırue la, valeur clficare, scule intéresssante pour les courants altermaluf;

J'ai constaté que duflérents trpes damperemilres, traversés, it la même fréquence, par le méme couranl pouvatent dnner ros erreurs de 10 pour 100 solon la forme de conibe du courant ; cola tient à ce que le rapport entre la valeur maximum et la valour effioace n'etait pas le meme.

Influence de la fréguence - Limtensido du conranl altruatif pour les voltmètros est donnce par la relation;

$$
\mathrm{I} \mathfrak{e}=\frac{E_{e n}}{\sqrt{R^{2}+L^{2}(1)^{2}}} .
$$

Il faudra done, pour avoir des mesures independantes de la fréquence, dimmuer le coefficient de self-minduction de la bobine at par conséquent la longueur des lignes fón force dans le far.

Il résulte, de tout ce qui prócede, que les indications de res: instruments peuvent être très aleatorres. el quil est somvent inecescaire ne tracer les gradualions avec le courant mime sur lequel do:vent servir ces appareilis.

() Bullelin de la Socielé inlemalionale des Electriciens, 190 
Action des champs extérieurs. - Les champs magnétiques extérieurs ont une influence encore plus grande sur ces appareils que sur les appareils à cadre mobile; la position des cables amenant le courant a souvent une grande importance. Cette action pournait a la rigueur être corrigée (par un nouvel étalonnement sur place), tandis que celle des courants passant dans les conducteurs voisins produit des erreurs accidentelles et irrigulières dont il est mpossible de tenir compte : Hartmann et Bram indique la distance de $50 \mathrm{~cm}$. entre l'équipage et un conducteur parcouru par un courant de 1000 ampères pour que l'action soit de 1 pour 100.

Boîtiers. - On peut remédier, en partie, à ces actions extérieures, par l'emplor d'un boitier en fonte, mais il ne faut pas oublier que l'hystérésis des boitiers peut jouer un ròle important; pour l'atténuer on disposera le solénolde intémeur de façon ì rendre minimum la longueur des lignes de force (dans le boîtıer').

Amortisement. - Ces apparells ont généralement un amortissement insuffisant ; c'est à eux surtout que s'applique ce que, je disais au début de ce rapport au sujet de l'amortissement.

Graduations. - L'action variant comme le carre de l'mlensité, les divisuons ne seront pas égales sur toute l'échelle.

Précision des mesures. - La précısion ne sera pas bıen grande, à cause de l'hylstérésis, etc. ; mais tout ce que nous pouvons demander, c'est d'avoir des indications concordantes. Une erreur relative de 1 à 2 pour 100 au 8 dizıèmes de la graduation maxıma sera bien admissible ; mass l'erreur absolue ainsi déterminée devra etre constante pour toute la partie utile de lichelle.

Wattmètres et compteurs. - Les wattmètres actuellement utılisés sont tous à lecture directe; on a renoncé aux appareils a torsion dont l'emploi était si pénible, et qui étarent inutilisables pour la haute tension. Les wattmètres servent surtout, pour les courants alternatıfs et doivent d'abord répondre à dıfférentes conditions faciles à rélaliser (dévıation proportionnelle à la puissance, amortissement parfait). Les causes d'erreur pouvant affecter leurs indications tiennent : $1^{\circ}$ Aux courants de Foucault se développant dans les parties métalliques de l'instrument ; $2^{\circ} \mathrm{A}$ la self-induction et à la capacité de la partie mobile et de sa résıstance en sérı ; $3^{\circ}$ Aux transformateurs ; $4^{\circ}$ A l'action des champs magnétiques voisins.

Courants de Foucault. - Les courants de Foucault, auxquels il faut joindre l'inégale répartition du courant dans les conducteurs (effet Kelvin) peuvent produine, dans le cas de courants très décalés sur la tension, des erreurs attergnant 10 et 20 pour 100 . On évitera ces actions secondaires, par la suppression de toutes les parties métalliques (boitte, support, etc). par le fractionnement et le toronnage des circuits. Tout mstrument mal construit à cet égard, devra etre rejeté.

Self-induction el capacité. - La formule de correction :

$$
P=P^{\prime} \frac{1+\operatorname{tg}^{2} \phi}{1+\operatorname{tg} . \varphi \lg . \Phi} \quad \text { avec } \quad \operatorname{tg} \cdot \varphi=\frac{j(1)}{R}
$$

indiquée par Flemming, suppose la non existence des courants de Foucault et de l'effet Kelvin dont je viens de parler ; dans cette formule $P$ est la puissance vraie, $P$ la pussance lue au wattmètre, $\boldsymbol{\phi}$ l'angle de décalage entre la tension et le courant dans le réseau, o l'angle de décalage entre la tension et le courant dans le fil fin. Cet angle est le seul sur lequel nous puissions avor action.

Les indications, non corrigées, sont le plus souvent trop fortes; mais le coefficient de correction sera d'autant plus farble que le rapport de $l$ coefficient de self sera petıt par rapport à $R$, résıstance du circuit dérivé. Cetle condition est bien remplie pour les appareils étalons, pour lesquels le coefficient $l$ est farble $\langle 0,003$ à 0,005 henry et $R$ grand ( 30 ohms par volt).

Quant à l'effet de la capacité de l'enroulement fil fin, on l'évitera par le fractionmement des résistances (a double enroulement et sans self) mises en série avec la bobine mobile.

Il est assez difficile, pour les compteurs d'énergie que $l$ soit petit devant $R$; aussi constate-t-on dans la marche de ces appareils, malgré les dispositifis compensateuns employés, des erreurs variables avec le décalage du réseau ; cette remarque est ımportante, car dans la mesure d'une puissance triphasée par la méthode des deux wattmètres, l'un des appareils peut fonctionner avec un courant très décalé sur la tension.

Transformateurs. - La question est encore plus dificile si le comptenr et le wattmetre sont munis de transformateurs de ten- sion, et surtout d'mtensilo; ces thasslormateurs viennent ajouter des décalages supplémentatres enllee less couranls primare et secondaire. Les transformateurs de tension, fonclionnant toujours avec la même induchion, seront relativement faciles à construme (la question d'isolement, seule, est importante) ; mais les transinn. matelurs d'mlensilé, dont l'mduchon est variable avec l'mtensilt' du courant, domnent lieu, bien souvent, à des erreurs graves.

Pour avor un rapport de transformation constant, les transformaleurs dorvent etre construits avec d'excellentes toles, avour une réluctante très fauble et être dépourvus de fute magnétıque $;$ ils seront done relativement lourds. Nous employons (*), au Laboritlosre central d'Electricité, pour les transformateurs d'untensifi des anneaux de toles circulaires sur lesquels les enroulements sont fartrs d'une laçon aussı régulière que possilnse. I La Sockèt? Sremens et Halske construt ses transformateurs de mesure sans jomts magnétıques, mais donne aux toles une forme rectangulair évidée. La seule difficultéi de l'anneau est le bobinage, mais ćest un fauble inconvénent devant le résultat obtenu. Les transforma. teurs a circuat magnítique ouvert, ou construits avec des joints, ne devraient plus être employés actuellement.

Actions extérieures. - Ces actions, dont il a été question pour les ampèremètres a cadre moble, causent, sur les indications des compteurs et wattmètres, des erreuns graves, car ces actions purement locales nexistent pas dans les verrfications des apparells alu laboratorre ; que de fors an-jc constalé une marche irréguhère de compteur, marche due, uniquement, à l'influence des conducteurs voisins ! Les compteurs, surlout à cause des queshoms pécunuares qui s'y rattachent, devratent etre éloignés, non siulement les uns des autres, mass encore de lout conducteur parconil par des courants intenses. Il ne faut pas oublrer que cortans types de compleurs, vendu comme astatiques, ne sont réellement aslatiques que dams des champs uniformes, cas rare dans les tableaux de distribution.

Erreurs. - Les erreurs, pour les wattmètres étalons construits d'une façon rationnelle, attemdnont au plus 0,5 pour 100 poul les 8 dixièmes de la graduatıon maxıma) ; mais, pour les comptenrs, clles sont beaucoup plus importantes; les frottements sur le: pivots, le décalage des courants, lemploi de transformateur, l'action de la température sur l'amortisseur en cuuvre (cette deruière produt 3 à 4 pour 100 pour $10^{\circ} \mathrm{C}$ ), fausseront les indications, ef selon la charge, et selon le type de compteur. Aussi, peut-on demander, en oourant constant, une précision de 1 it 2 pour $100 \mathrm{et}$ cela senlement à partur de un dixieme de la purssance tolale di compteur.

En régine variable, sur les réscaux de traction et de transport de force, la mesure exacte de liénergı est un problème encore à résoudre; 11 n'est pas rare de constater sur les compteurs des erreurs de 10 a 20 pour 100 , erreurs varables d'un instant ì l'autre avec le sens des décalages du courant dans le réseau.

Électrodynamomètres - Les électrodynamomètres à torsion (genre Siemens) ne s'emplorent plus mantenant; mans les balarces bien connues de lord Kelvin sont toujours considérétes comme d'excellents appareils étalons ; elles ont cepiendant l'moonvénient d'ètre insuffisamment amorties ; de plus, dans les grandis molèles (kilo-aurpères), où les circulis sont formós de barres massives, les inducations peuvent etre mexactes em courant alternatif. L'erreur atlent 6 pour 100 avec la fréquence 40 , et vare avec elle.

On construit actuellement des électrodynamomètres à lecture directe servant de voltmètres ou d'ampèremètres. Ces instruments ayant un champ magnétıque intérıeur très faıble, leurs indications seront faussées par les champs extérieurs ; le champ terrestre, par exemple, peut produire une erreur de 2 pour 100 ; aussi, dans le cas d'emplo pour les courants comtinus, faudratll faire deux lechires en inversant le sens du courant.

L'intensité nécessaire, pour la déviation totale, peut varier de 0,5 i 0,05 ampère ; elle dépend de la tension pour laquelle l'apparell a été construit.

Lo.j voltmètres, à partir de 100 volts, ont leurs indications sensiblement indépendantes de la température et de la fréquence.

Less ampèremètres, dont la bobine moblle ne pent supporter le courant total, ont cette bobme mobile en dérivation sur le circuit fixe. Dans ce cas, pour que l'étalonmement soit le meme en coutrant continu et alternatif, il faut que la constante de temps soit la même pour chacun des circuits et, par consćquent, que la

(") JaNeT ET ILIovm, Bulletin de la Société internationale des Eleclriciens, février 1903. 
résstance $R$ ne varie ni avec lá lempérature ni avec l'mtensité du courant. On esl donc amené à admethre une densité de courant assez fauble dans les enroulements.

Electrometres. - Ces appareds consommant peu de courant, sont employés pour les hautes tensions. Les forces mises en jeu sont lrès labbles, aussi la construction est-elle délıcate. Les mdications peuvemt varier sous l'influence des champ's électroslahques (rottemenl sur la cage, etc.) et des étmcelles qui se produisent souvent entre l'argulle et les plateaux. On recommande, dans le bul deviter la détéroration de l'instrument par l'étncelle entre platenux, d'intercaler des résistances lıquides sur le trajet d'un dus conducteurs ; ce clisposituf est excellent à condition de ne pas employer une rilsistance trop grande ; et ln est toujours prudent do vénfier que cette résislance ne trouble pas les indications de l'apparel. J'al conslaté des erreurs grosislères dues à des contacts nux bornes insuffisamment décapés ; il faut donc surtout avec ces instruments, avoir des fils de connexion excellents. Lisolemont des dectrometres peut etre compromis après quelques jour de services par l'ozone qui se dégage.

Ces appareils indıquemt les valeurs efficaces, mais leur emplor est limité par la tchision maxima de londe ; c'est cette tension maxıma qui dort déterminer l'écart entre l'aıguille et les plateaux ; telle est la rasson pour laquelle on vout les apparenls vendus pour 50.000 wits, itre inutilisables it partir de 35.000 volts efficaces. (hin se lrouve donc amené pour les hautes tensions, ou à employer des apparels volumineux ou à se servir de réducteurs de potenliel : ces róducteurs peuvent être constıtués par une résistance, sans soll ni caprecité, aux extrémités de larquelle est applıquée la teisson à mesurer ; l'électromètre à basse tension est mis sur une fraction connue de ces résistances. Au lieu diemployer ces n'tintances qui absorbent de l'énergıe, on peut se servir de capaciths mises en série, l'électromètre sera branché en dérivation sur une des capacités : mais ıl faudra, pour cela, que la capacté de lélectromètre solt néglıgeable devant la capacité ct.alon ; il faudra, (4) outre, que les diélectriques de ces capartís ne se modifient par sous l'mfluenco prolongée de la tension.

Deqré de précision. - - Dans ces apparesls, les divisions ne sont pas égales, mais l'inégalité peut elre corrıgée, en partie, au moven d'un procédé mdıqué par Ayrton; 11 suffit pour cela d'mtercaler sur le trajet de l'álectromètre une capacté convenable. Quelle que soit la méthodle employée, il ne faut pas compler, pour les électromètres a haute tension, sur une exactstude de plus de 2 à 3 pour 100 ; les électromètres à basse tension peuvent etre exacts à 1 pour 100.

Appareils d'induction - Les ampèremètres, voltmètres, waltmètres d'mduction, dérıvent tous du proncre indiqué par Ferrarıs : ces appareils, si employé a lheure actuelle sur tous les tableaux alternatıfs, sont très robustes, mais malheureusement leurs mdications dépendent de la fréquence et de la forme de rourbe du courant alternatif ; lls subissent aussi linfluence des champs magnétıques extémeurs, mass dans une assez fable mesure, car pour leur fonctionmement lis disposient de champis intérieurs très intenses. Les variations de température peuvent produire des erreurs de 2 à 3 milhèmes par degré centigrade.

Les transformateurs sur lesquels ils fonctionnent d'habitude revront avoir les memes qualités que coux des wattmètres.

Appareils thermiques. - Les apparells thermiques genre 'ardew, actuellement disparus, ont fast place à d'autres dans lesquels une petite portion du fil est dulatée par la chaleur dégagíe sous l'influence du courant. Les appareils Hartmann el Braun sont les plus connus et de beancoup les plùs répandus.

La température du fil est souvent si b́levée qu'll ne peut supporter sans se rompre une surcharge relativement faible. Tous ceux rui ont employé les thermiques savent avec quelle napidıté arrive la rupture du fil, et cela malgró les fusibles dont ils sont muns.s par le constructeur.

La chaleur dégagée produit, malgré les dispositifs compensateurs employés, des déformations du support du fil amenant des déplacements du córo. L'mindécision de lecture qui en résulte peut être importante.

Les aiguilles des thermiques prennent lentement leur position dequilibre ; leuns indications seront donc en retard ou en avance sur les valeurs vraies; cela a peu d'importance, en général, et vient contribuer à l'amortissement.

Les raleurs indiquées sont indépendantes de la forme de l'onde, de la fréquence et des actions extérieures, mais la fragilité de ces instruments les fera remplacer, de plus en plus, sur les fableaux, solt par les appareils d'induction, sout par les électromagnétıques bien étudiés.

La consomnation des themuques est très élevée, environ 0,2 à 0,3 ampère pour les voltmètres et a 0,2 volt pour les ampèremetres, ce qui oblige it employer, pour les hautes tensions, des transfurmateurs.

Montage. - Il faut, pour de: courants supérieurs a 300 ampères, torsader les fils reliant le shunt au voltmètre de mesure, car le courant principal peut produre des forces électromotrices suffisant d'maluction pour fausser les indications de 1 à 2 pour 100 . Il est aussi essentiel de ne pas modifier la nésistance des cordons somples et de leurs attaches. La précision des mesures est de l'urdle de 2 pour 100 pour les 8 dizı̀mes de l'échelle.

Appareils enregistreurs. - Les systemes de mesure des enreglstreurs sont construls sulvant les différents principes que nous avons examinés; 1 ls en ont par conséquent tous les inconvénent ; ll faul ajouter à ces défauts les irrégularités produites par le disposilif enregistreur lui-meme ; aussi ne peuton attendre de liurs indications que des résultats souvent grossiers.

L'enregistrement se fast d'une façon continue ou discontinue Dalus le premrer cas le frottement de la plume ou de la molette sur le papier produt, le plus souvent, un tracé défectueux; l'enr. gistrement discontmu est obtenu par pointe d'une algulle, $n$ par percement du papier (enregistreur à étincelle de Siencni-) pour les deux cas, les variations rapudes ne sont pas enregist:" et seronl fausisées par l'oscillation propre de l'appareil. A avis, al faudrait comme pour les oscillographes, avoir une granki force directmce et un petut moment d'mentie ; cette demiere co: chtıon, difficlle d obteniır avec des apparells à alguille, serait ré:l. sable pain l'enregistrement photographique des déviation d'ı rayon lummeux.

L'enregistreur oscillographique rendrait les plus grands servic. potr la mesure de la puissance et l'étalonement des compteurs en rigime variable.

Fréquencemètres et synchroniseurs. - Les fréquencemètres Hartmann et Braun basés sur la résonance de lames vibrantes le synchroniseur Limroln, et celui d'Everett (à champ tournant) sont très employés.

La Compagnie pour la fabrication des compteurs construit un fréquencemètre basé sur l'action de deux circuits attrant deu. noyaux de fer solndarre de l'aigulle. Les deux circuits sont relı: en dérivatron l'un sur l'autre, mas un des circuits est complété par une rósistance sans self, l'autre par une bobme de self. Une bobme de self-manction, mise en sérle avec lensemble des deux bobmes, est destmée à ćtoulfer autant que possible les harmomuques. La déviation de l'argulle dépend du rapport entre les conranis dans les bobmes; quand la fréquence est faible il passe beancoup de courant dans la bobme avec self, et son noyau est tortement attiré, l'inverse a heu si la frúquence augmente.

Phasemètres. - Le seul moyen d'avoir, exactement, la dilfk rence de phase entre deux courants est d'employer une méthode oscillographique ; culte méthode montre que, sauf pour le cas rare do comrants sinusoldaux, la dilfírence de phase entre les deux maxima est différente de celle des deux passages ì zéro; dans ces conditions, les appareils industrels ne peuvent servir qü̈ titre de renseignemenl, leurs indications se rapportant à langle de décalage ficlif $\infty$ qui existerait entre deux courants smusoidaux ayant ce même dúccalage.

\section{III. - APPAREILS DE LABORATOIRE.}

Dans cette revision très rapide, nous n'examinerons que les mincipaux apparesls.

Boiles de résistance. Ponl de Wheaststone. - Tous les contstructeuns emploient maintenant, pour les bobines, le manganin. Les résistances ont ainsi un coefficıent de température négligeable, mais 1l est nicessare, pour avour une valeur stable, de faire viellir, artificiellement, lalliage.

Les fiches des boltes laissent beaucoup à désirer ; leur forme comique n'assure pas toujours de bons contacts et devrait etre remplacé par des serrages plats facilement accessibles et d'un netloyage commode. La maison Carpentier est entrée dans cette vore, mais seulement pour les ponts de haute précision.

Le vernis dont on enduit les résistances est souvent hygrométrique et a une influence variable d'un jour a l'autre, influence d'autant plus grave qu'elle peut passer inaperçue.

Les boites à résistance en série devraient être remplacées par 
les boiles a dicades ; je sigmale le montage Nugues-Carpenticr comme ayant los avantages des boîtes à dccades, tout en employant mons de bobrries.

Il ne faut guère compter, malgré tout ce 'qu'on assure, sur une erreur inferieure au millieme (en valeur absolue) ; et c'est seulement avec des précautions lout ì fart spéciales qu'on pous ra attemdre une préession plus grande. Cette errcur allectera, bien entendu, tous les méthodes et apparenls de mesure dans lesquels enlrera la valeur vrale des résistances et non leur rapport.

Ront double de lord Kelvin. - 10 Modèle mdustrel, dans lequel Ia valeur de la résislance est comparée à une longueur pise sur une barre étalonnée. La barre étalcm devra otre bien calibrée et bien homogène comme résistance lméare. L'erreur relaluve est variable selon la position du curseur sur la barre et il y a, toujours, incertitude, non seulement four la position d'équlibre, mais encore pour la valeur absolue de la longueur.

$2^{\circ}$ Modèle de précision dans lequel la résistance étalon csi fixe, mais où la mesure s'effectue par la variation du rapport dos bras de proportion. On trouve des ponts montés de cette manière, mais qui ont, à mon avis, dans leuns bras de proportion des résistances trop faibles ; ces résitances ayant quelquefols $10 \mathrm{ohms}$, des contactis mal assurés, ou môme les fils de connexions allant à la risistance ì mesurer introduiront facilement des erreurs de 1 pour 100.

Résistances élalons pour fortes intensités. - Ces résistances, construtes autrefois en mallechort, sont établies mamienant en manganin. Une élévation de température de $100^{\circ} \mathrm{C}$. prodult une vallation de $\mathbf{4}$ centièmes sur la valeur d'une résistance en malllechort, tandis que pour le mangamin la variation est dix fois mondre ; ll faut donc, avant tout, éviter une température élevée qui, outre la variation ci-dessus, produrat un changement dans la constitution de l'alliage. La grosse dificulté pour ces rísistances est de dissiper la chaleur dégagée par le courant. plusieurs moyens sont employés :

to Le refroidussement par laur ; c'est le mellleur, mais ll entraine à une grande surface de refroidısisement et à une masse de métal souvent constdérable, de sorte qu'il est mpossible à uthliscr si l'on veut rester dans les limites de températures acceptables.

$2^{\circ}$ Le i efroidissement par l'hule de pétrole. Ce dernier disposituf, étudié par la Reichanstalt, semble donner d'excellents lisultats. Les lames de manganm plongent dans du pétrole qui, dans ce cas, est refroidi par un courant d'eau passant dans un serpentm ; il y a toutefois lieu de craindre une altération du mamganm par le pétrole (pluss ou moins acide) ou par. l'eau dans le cas de fuitas du serpentin : il ne faut pas oublier que le manganm est très altérable par lhumidité.

$3^{\circ}$ Le refroidissement peut etre oblenu en fansant passer un courant d'eau dans la résistance étalon constitucie par un tube creux. Ce modèle, très séduisant par son enombrement minime, ne peut être faut en manganin. (qui serait altéré par l'eau) ; j'al constaté pour le mailleclsort que la résıstance augmentait avec re temps, ce qui semble dù à une atlaque ou à une usure du tube par l'eau.

Ohmmètres et vérificateurs disolement. - Sous ce nom on trouve dans le commerce des appareils basés sur des princrpes ditrérents :

$1^{0}$ Les uns, composés d'une ballerie de piles et d'un galvanomètre indwquent, par la déviation de l'aiguille diu galvanomètre, la valeur de la résistance mesurée. Les mdıcations de ces appareils sont faussées par les champs magnétiques extérieuris et par la variation de la difirtuence die potentiel de la pile ; co qu'il y a de facheux c'est que toute diminution de celte différence do poténtiel donne, pour la resistance a mesurer, une valeur trop grande (l'erreur peut attemdre 50 pour 100). Il est donc de toute ulilité de controler, souvent ces apparenls ; a cet ellet, certains d'entre eux comportent une résistance étalon permettant de vérifier un point de la graduation, ou sont munis d'une magnéto qu'on fait tourner à une vitesse déterminćte Sur le même principe sont construits des appareils fonctionnant avec la tension du réseau ; linfluence de la pile est ainsi évitée.

$2^{\circ}$ D'autres sont constitués par des ponts de Wheatstone.

30. Enfin les ohmmetres Evershed et Carpentier, basés sur une disposition indiquée par Maxwel, permettent d'élminer l'erreur due à la vamation de la force éllectromotrice.

Dans toutes les mesures d'isolement, on sait qu'il est nécessaire de fame les essais sous une tension voisine de celle du réseau, car les résistances d'isolement varient avec la tension ; celte observation doit guider pour le choix de l'appareil à emplover.
Piles élalons. - L'élément Latmmer Clark n'est pills nsité a cause de la variation de sa force ćlechomotrice avec la tomb. rature (1 millivolt environ par degré) ; au conlrare, les blomenl Weston, sensiblement indépendant de la lempiralure (dans cor tames limites), sont universellement aloptés. La force électro motrice de lísément Weston est d'environ 1,0185 volt (a) 2010) $\mathrm{L}$ a détermination de la valeur exacle de cet élément est, (n a moment, le sujet de très importants travaux de recherches dan les principaux laboratoires scientifiques du monde of en Francis à la Sorbonnie, au Laboratorre central d'Electricillé.

Les produts purs du commerce sont insuffisants pour la cols. truction des étalons au cadmium (") ; aussi faut-ll avor recours it des prodults préparés et tratés suivant certanes millodes: c'est à cette condation que les forces électromotrices des étalon: dí divers constructeurs ne dilfèrent pas de 1 clemi millıvolt pour in mème températule.

Potentromètres. - Les dispositıfs potentıomélruques se 3011 dúveloppés beancoup dans ces deruières annces; inas la lenteur des mesures fera toujours du potentiomètre un insirument de labonatoire. Ces apparels, destués ì des détermmalions mécisp exigent des courants très conslants, anssi est-rl de lonte néces sité d'avoir, pour le circuit potentiométrıque proprement dit, un courant ne dépassant pas un millicme d'ampère. Avcc cette batensitie, on peut employer comme source auxilialre une prle (pule de macrophone par exemple), tandus que, pour les mudèles exigeant un courant plus mitense, ll sera nécessaure d'avour cos accumulateurs et de vérifier constammentl le ríglage du corcunt du polintiomètre. De plus, avec un conrant fort, les défauts de conlarl (notamment les contacts glissant sur fil) prendront une grande importance et seront une source de varialions of dicmuss.

Elalons de self-induction el d'induction mutuelle. - 11 est tris dıfficıle de dóterminer la valeur d'un ćtalon de self-induction; les diffirentes méthodes employces ne permettent pas de compter sur ume précision supérieure à 1 pour 100. Les ótalons d'induction mutuelle, au contraire, peuvcont ettre calculés, a condilion tontefous qu'ils soient dépourvus de fer ; l'exactitude clo scr's étalons dẹpendra de la précisuon avec laquelle saront díterminces leurs dintit. slons géométriques.

Oscillographes. - Ces apparels sont des plus précieux pour l'étude des courants électriques valrables ; mais ils sont cncure. malheureusement d'un mansement délicat et il serast a souliaitur quiune construction plus parfaite rendit leur emploi plus fartle Le système oplique, en générral défectuenx, ne permet pas d'avor une bonne image photographique des courbes.

ondographe dilospitalier. - Cet apparen ne permet poin. comme les oscllographes, d'avour la courbe corresfondant it li même période ; il peut rendre nóammoms des services quand ॥ s'agit d'une succeission de phénomènes pérıodıques idenliques.

Ilystérésimétres. - L'apparesl d'Ewing, qui eut jadis u!p grande vogue, est dujourdhus presque abandonnć. Cet appareil présente de nombreux défauts. Linductıon utilisće, 4000 gauss envinon, est beaucoup trop faible, surtout à l'heure actrelle oit l'on fabrıque des toles dans lesquelles les pertes en fonction de l'mduction croissent beaucoup plus vite que ne l'molique la bi de Stenmetz. L'éprouvette utalisće est d'une masse beanconm Irap fable, ćtant donmées les grandes variations que présente lo mítal survant les points de la tôle oì l'on a prélevé l'ópruuvelle ct suivant que cette éprouvette a sa grande dimension parallèle on perpendıculaire au sens du lammage. De plus, les phénomènes ilu vienllissement rendent peu sur l'emploi diópronvettes à falbles pertes.

L'hystérisımètre Blondel est aujound'hui plus employé Cet appareil qui utllise une induction denviron 10.000 Gauss présent? quelques inconvénents : masse de l'éprouvette frop faible, mesurt de perte par hylstérćsis ne correspondant exactement ni à l'llystít résis tournante, ni à l'hysténésis altematıve.

La váritable méthode pour la détermmation industrielle des pertes par hystérésis est la méthode préconisée par l' $\Lambda$ ssoctation des Ingénieurs electriciens allemands : détermination par la méthode du watitmètre des pertes totales, pour l'ınductıon 10.000, la frítquence 50 et la tempénature de $30^{\circ} \mathrm{C}$; on emplore dans ces conditions des éprouvettes ayant environ une dizame de kilogrammes.

Le procédé qui consistemant a decouper, pour faire cette mosure, des éprouvettes constituées par l'empilement de rondelles circl.

(*) Lls peuvent produire des différences de 2 millièmes environ. 
laires presenterail des difficulles de bobnage auxquelles on ne

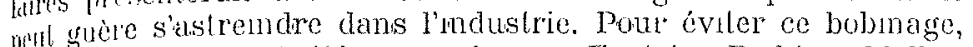
dyers appareils ont ćté proposés par Epstein, Rıchler, Mollmderer ( $\left.{ }^{*}\right)$. D'après Gumlich et Rose, ces apparouls donnent des résulger (1ui ne dillerent que de 2 i 3 pour 100 des résultals foums par lambau bobmés ; aucun d'eux n'a pourtant encore reçu lapprobatum offictelle de l'Assoctation des Jisectriciens allemands. Il ne faul pas oublier que celte méthode nécessite, pour donner des résultats, un certam nombre de mécautions qui restresgnent saurent son emplor dans l'industrie.

perméamètre. - Les anciens types de perméanèlres, dHopknson, à árachement, sont aujourd'hui presque complètement abanfunnís, du moms en France. L'apparesl de Kath esl encore assez

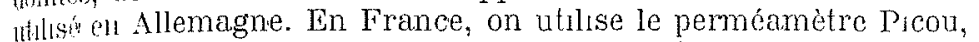
anstrul par Carpentier. Cet apparel semble donner de bons wisulkals, au moins dans les mduchons moyennes. Les valours truvées pour les faubles inductions sont évidemment altérées par l'nunos'sbultés ou l'on se trouve de désammanter complètement lo fer. Il semble, d'après des essaus falts au Laboratore central dElectristic, domer aux inductions élevées des valeurs 10 pour 100 phis faibles que les valeurs vrajes. Cela tient probablement, comme la signadél Gumlich, à l'erreur causéc par l'oblıqulé des lignes de force dans les joints.

Il est bon de ne pas oublier également que les phénomènes de viscusité magnétıque peuvent dans le cas d'acier très doux fausser grarement les résultats sur des barrettes d'acıer massif.

Pour les très hautes inductions, de l'ordre de celles qu'on renconitre dans les dents de dymamo, la maison Carpentier construst depus quelque tempis un nouveau perméametre $\left.{ }^{\star \star \star}\right)$, dans lequel ripronvetle ne constitue plus un crrcuit magnétıque fermé, mais se compose d'un barreau droit. Un dıspositıf ingénieux apporte inumblatement la correction due à l'existence du champ démagnćIsant Linconvínient de cel appareil est d'admeltre la constance in facleur démagnétisant de du Bors. Cet appareil est encore peu répandu et il est difficile de se prononcer sur la valeur des résullals filil foumit. Nous ne pouvons qualtirer l'altention sur l'ntúrếl qu'll y a à étudier los tôles dans ces réglons de salurathon.

Bomons-nous à rappeler que, dans les laboratoires, la perméaInlté el les pertes par hystérésis-statique se mesurent par des délermmations faites au moyen du galvanomètre balıstique, sur des ćprouvettes en forme d'anneau.

\section{EXPOSITION DE MARSEILLE}

\section{APPAREILS DE MESURE}

La maison Chauvin et Arnoux exposait divers appareils de mesure pour tableaux de distribution ou pour contróle, notamment des galvanomètres, ampèremétros et milli-ampèremètres, voltmètres et milli-voltmètres, wattmètres ordinaires ou enregistreurs, pour courants continus ou alternatifs, à haute ou basse tension: des ohmmètres pour mesure d'isolement, une caisse portative pour la mesure des hautes résistances, un pont de Wheatstone à décades, des galvanomètres différentiels, des voltmètres compoundés donnant le voltage à l'extrémité des feeders sans l'intervention de fils pilotes.

Signalons aussi un volt-ampère-wattmètre calorique permettant, par le simple déplacement d'une connexion, la mesure directe de la tension, de l'intensité et do la puissance d'un circuit parcouru par des courants continu ou altcrnatifs. L'appareil se compose d'un fil A CD B, disposć en deux brins parallèles, s'enroulant sur un cylindre $C D$, et dont les extrémités A et B sont fixes (fig. 1). Le crylindre, fixé à une lame flexible DE, et sollicité par le ressort IF, oscille autour du point E lorequ'un des fils AC ou BD s'allonge. Cette rotation est amplifiée par un levier commandant l'axe do l'aiguille indicatrice de l'appareil, au moyen d'un fil de cocon tendu par un ressort additionnel. L'index dócrit

(") Volr à ce sujet Gumlich ol Rose E.-T.-Z., 1905, p 408 ol 503 .

(") H. Arat donat, Revue eleclrique, 28 févmer 1907 . des angles proportionnels à la différence d'allongement des deux fils identiques $\mathrm{AC}$ et $\mathrm{BD}$, de sorte que les variations de la température ambiante n'ont aucune action sur l'aiguille de l'appareil.

Pour mesurer la tension, les bornes $a$ et $c$, correspondant au fil dilatable $\mathrm{AC}$, sont reliées aux deux pôles par l'intermédiaire d'une bobine de circuit (représentée à la partie supérieure de la fig. 2). La valeur de la tension s'obtient en multipliant le chiffre $D_{t}$, lù sur l'échelle des volts, par la constante $K_{1}$ poinconnéo sur la bobine du circuit.

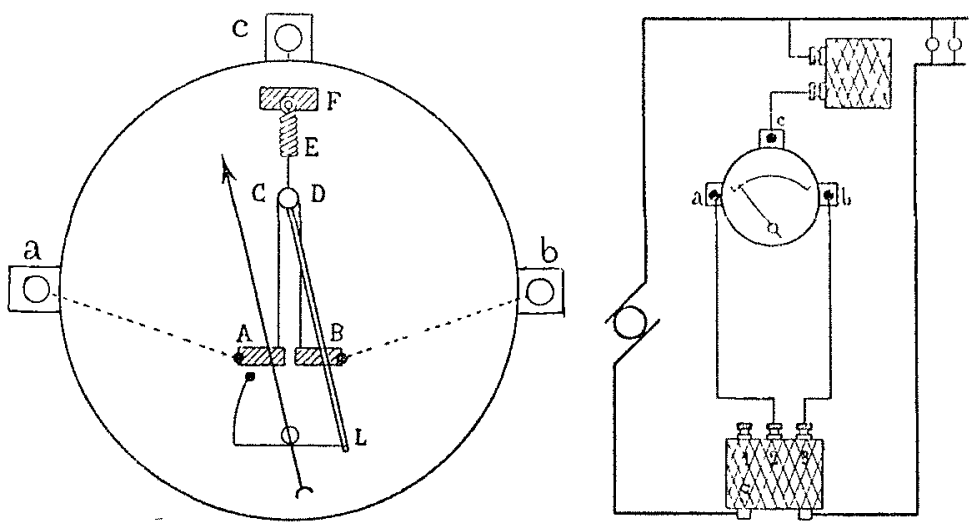

Fig 1 et 2. - Volt-ampère-waltmèlre hermique.

Pour la mesure de l'intensité, les hornes $a$ et $c$ du galvanomètre sont reliées aux hornes 1 et 3 du shunt $\mathrm{S}$ intercalé dans le circuit à mesurer. Celle intensité est égale au produit de $D_{a}$, lù sur l'échelle des ampéres, par la cons. tante $K_{2}$ poinçonnée sur le shunt.

La puissance est égale au produit $K_{1} K_{2} D_{\|}, D_{\text {w }}$ ctant lù sur l'échelle des watts, les bornes $a$ el $b$ du galvanomètre étant reliées aux bornes 2 et 3 du shunt.

La maison Cadiot, dépositaire en France des apparcils IVeston, exposait un certain nombre d'appareils de mesure de précision, notamment des voltmètres et ampèremètres à compensation, dans lesquels les mesures sont rapportécs à une résistance constante et à la force électromotrice d'un élément de pile Weston. Ces appareils ont été décrits daus L ( Houille Blanche, d'aoûl $1908\left(^{\star}\right)$.

La Compagnie pour la fabrication des Compteurs exposail toute une séric de ses appareils de contrôlo, types tabliau ou étalon, du système Meylan-d'Arsonval, enrégistreurs ou non : voltmètres et amperemètres à aimant pour courant continu, thermiques ou électromagnétiques amrort is pour courants alternatils, wattmètres électrodynamometres ou d'induction. Elle exposait aussi des compteurs : système Thomson, à 2 ou 3 fils, pour courants continu ou alternatil", système $\mathrm{O} K$ à 2 ou 3 fils pour courant continu, systeme A.C.T. pour courants alternatif's mono ou polyphasé, cos deux derniers systèmes du type ordinaire ou à prépaiement préalable (disposilif Berland), système à double taril ou a tarıf multiple, ou à dépassements.

Tout le monde sait combien est grande, suivant les heures de la journée, la variation de la charge des usines génératrices des réseaux de distribution d'éner gric électrique. Par suite de cette grande variation, of du passage des pointes, ces usines sont obligées de disposer d'un matériel supplémentaire qui ne travaille que trés pou de tomps, et est par suite fort mal utilisé. Il est donc logique de faire paycr plus cher l'énergie électrique qui est fournie par cos machines supplémentailes, et moins cher celle qui est fournie par les machines fonctionnant continuollement à pleine charge, c'est-à-diro à leur maximum de rendement. Ce mode de taxation est rendu possible par le dispositif de

() La nuason Cadiol exposat ausst des jroduits isolants de MM. Parge et Cir de lierlin. Elle montrat des induits de dynamos, dont les fils du bobnage elarent isoles avec le matiol l'aege. 